\title{
Airport Connectivity Optimization for 5G Ultra-Dense Networks
}

\author{
Saba Al-Rubaye, Senior Member, IEEE, and AntoniosTsourdos
}

\begin{abstract}
The rapid increase of air traffic demand and complexity of radio access network motivate developing scalable wireless communications by adopting system intelligence. The lack of adaptive reconfiguration in radio transmission systems may cause dramatic impacts on the traffic management concerning congestion and demand-capacity imbalances driving the industry to jointly access licensed and unlicensed bands for improved airport connectivity. Therefore, intelligent system is embedded into fifth generation (5G) ultra-dense networks (UDNs) to provision dense and irregular deployments that maintain extended coverage and also to improve the energy-efficiency for the entire airport network providing high speed services. To define the technical aspects of this solution, this paper addresses new intelligent technique that configures the coverage and capacity factors of radio access network considering the changes in air traffic demands. This technique is analysed through mathematical models that employ power consumption constraints to support dynamic traffic control requirements to improve the overall network capacity. The presented problem is formulated and exactly solved for medium or large airport air transportation network. The power optimization problem is solved using linear programming with careful consideration to latency and energy efficiency factors. Specifically, an intelligent pilot power method is adopted to maintain the connectivity throughout multi-interface technologies by assuming minimum power requirements. Numerical and system-level analysis are conducted to validate the performance of the proposed schemes for both licenced macrocell Long-Term Evolution (LTE) and unlicensed wireless fidelity (WiFi) topologies. Finally, the insights of problem modelling with intelligent techniques provide significant advantages at reasonable complexity and brings the great opportunity to improve the airport network capacity.
\end{abstract}

Index Terms-Airport connectivity, Fifth generation (5G) Energy efficiency, Load balancing, Network coverage, Power optimization, Ultra dense networks.

\section{INTRODUCTION}

$\mathbf{T}$ HE rapid increase in traffic load and the requirement for ubiquitous access network services in airport domains have triggered a dramatic expansion of radio access infrastructure that subsequently led to a huge increase in consumed power. The fifth generation $(5 \mathrm{G})$ networks, specified as International Mobile Telecommunications-2020 (IMT-2020 Standard), support multigigabit downloading speed in few milliseconds latencies to connect various types of services and machines [1]. Although 5G addresses a wide range of use cases, there is no standard or globally agreed radio interface that defines a dedicated data communication system for simultaneous airport operations or airport connectivity. There are different commercial systems (e.g. cellular solutions, custom/proprietary systems (Gatelink, etc.)) that are in operation at various airports, however, they provide a limited capacity compared with the rapid increase in the number of connected airport machinery that increasingly become a bottleneck of the air transportation network [2]. There is also the problem of compatibility since most of current technologies are meant to connect users and Internet of Things (IoT) sensors. These are not used for air traffic management (ATM) communication due to the issues of reliability, availability, and security. This marks a strong need to incorporate additional services within the current airport infrastructure such as Air Traffic Control (ATC) communications, very high frequency (VHF), Aircraft Communications, Addressing and Reporting System (ACARS) and Satcom Global (SATCOM) systems. For air interface, the VHF has limited spectrum and is already fully occupied with heavy traffic congestion and does not have the capability to support the rapidly growing air traffic and future highspeed data applications, while SATCOM is costly and does not provide good connectivity at the airport surface due to interference and buildings. To this end, there is a need to develop a new radio interface that connects all users within airport domain.

The evolvement of artificial intelligent (AI) techniques can improve the wireless access by intelligent learning about the spectrum availability to assign data within the existing radio capacity. Following the new approach of intelligent system can improve the resource utilization and potentially support proactive decisions on radio access actions. To elaborate on airport technology features, the emerging ultra-dense networks are anticipated to be a combination of multi-tiers, radio access technologies with different transmission rate and coverage sizes [3]. Therefore, low-power small cells deployments within macrocell layout are key components to enhance the energy efficiency for the entire airport domain. The LTE Release 15 introduces a new features for cellular network, to improve the coverage and enhanced system capacity, which makes significant development in energy efficiency that needs to satisfy challenging requirements for supporting several functionalities [4]. The carrier aggregation technology is a key feature to boost LTE capacity by aggregating multi-carriers to support higher speed downloading at the user side [5]. Therefore, wireless networks are expected to have more dense structure than before [6], consisting of operator overloaded by small cells (e.g., femtocell, pico, and micro) to emerge as usersdeployed units that can be governed by macrocell to improve service delivery and threatens to increase the networks energy efficiency. LTE is expected to be a main part of next-generation aviation communication system to support fixed and mobile services for aircraft surfaces. In the 5G UDN paradigm [7], it is more reasonable to find alternative ways to exchange 
data rather then fibre networks, which was the case in the old infrastructure, partially because the high cost of deployment. As an alternative, networks may employ millimetre wave technology to deliver the data through short-range highcapacity wireless links. Consequently, dense network of small cells are becoming the primarily solution to deliver traffic to end users over less-occupied unlicensed channels. However, this type of network infrastructure consumes a lot of energy that can be reduced through energy-aware resource allocation schemes. The optimization strategies to minimize the total power consumption and improve energy efficiency are either employed by site provisioning entities to reduce the number of operational base stations or by deploying small cells that consumer lower power [8], [9]. To elaborate, the challenge of power efficiency in dense networks scales-up when deploying more small cells [10]. Therefore, pilot power utilization for energy consumption may become a less effective method in small cell networks compared with other optimization schemes that tackles the network infrastructure. Therefore, we focus on a new energy optimization scheme that considers multi-tire of base stations in $5 \mathrm{G}$ UDNs.

\section{A. Related Work}

Recently, several noteworthy studies has been considered intelligent solutions [11], to facilitate the network operators, while reducing the overall complexity and simplify the reconfiguration procedures of mobile networks. The main objective of the existing approaches is to leverage decision making for an airport based macrocell to transmit data with very low power to support multiple end users (e.g. aircraft), considering the various conditions of traffic load within the cell. An optimization solution based intelligent approach will extend the coverage area and improve the system capacity in $5 \mathrm{G}$ UDNs leading to new models of intelligent network coverage that can support a large number of users with high data rates [12]. Some of optimization techniques have been successfully applied, for example in [13], the convex optimization problem has been considered to solve the power consumption problem by maximize energy efficiency while satisfying the system requirements. The pilot power transmission scheme in [14] investigated an intelligent network coverage model according to the aircraft location. Furthermore, the maximization of energy efficiency in LTE macrocell is formulated as an optimization problem for each base station power constraints. In [15], the near-optimal algorithm achieves energy efficiency performance with low complexity. However, these studies do not consider the impact of the dynamic changes in traffic on the network power consumption.

The authors of [16] considered a power optimization model for load balancing and show that network performance can be enhanced by proper adjustments of the pilot powers. However, the authors do not consider the impact of small cell deployments. Furthermore, a stochastic optimization problem is considered in [17] to mitigate the effect of cell outage and provide efficient cells deployment. In [18], the authors considered optimization technique using mixed integer non-linear problem (MINLP) to improve the wireless throughput and
TABLE I

List OF MAIN VARIABLES AND PARAMETERS

\begin{tabular}{|c|c|}
\hline Symbols & Definitions \\
\hline$m=1, \ldots, M$ & Cells in the system \\
\hline$k=1, \ldots, K$ & Set of base stations \\
\hline$j=1, \ldots, J$ & Set of vehicles \\
\hline$G_{k j}^{d l}$ & $\begin{array}{l}\text { Downloading power gain from base station } k \text { to aircraft } \\
j\end{array}$ \\
\hline$P_{k}^{t o t}$ & Total power transmitted from base station $k$ \\
\hline$P_{j}^{t o t}$ & Total power transmitted from aircraft $j$ \\
\hline$P_{k}^{m x}$ & The maximum transmission power \\
\hline$\eta_{k}^{d l l}$ & Cell load factor \\
\hline$P_{k j}^{p c h}$ & $\begin{array}{l}\text { Amounts of power devoted to a pilot channel from base } \\
\text { station } k \text { to aircraft } j\end{array}$ \\
\hline$I_{k j}$ & Total interference between base station $k$ and aircraft $j$ \\
\hline$\delta_{d l}^{p c h}$ & $\begin{array}{l}\text { Portion of interfering power from the primary cell power } \\
\text { mainly due to multipath signals that are not orthogonally } \\
\text { captured by the terminal user receiver }\end{array}$ \\
\hline $\mathcal{N}_{j}$ & Thermal noise power at aircraft $j$ \\
\hline$\Gamma_{k j}^{p c h}$ & $\begin{array}{l}\text { Received signal to interference density }\left(E_{c} / I_{o}\right) \text { ratio } \\
\text { for aircraft } j \text { from base stations } k\end{array}$ \\
\hline$\rho_{j}$ & $\begin{array}{l}\text { Threshold sensitivity required to detect a signal at } \\
\text { aircraft } j\end{array}$ \\
\hline$C_{k j}^{d l}$ & $\begin{array}{l}\text { Capacity of a link between base station } k \text { and aircraft } \\
j\end{array}$ \\
\hline$\beta_{k}$ & Frequency bandwidth of base station $k$ \\
\hline$C_{j}^{d l}(t)$ & Downlink capacity for each at a specific time $t$ \\
\hline$P_{j}^{p c h}(t)$ & Power received by each aircraft at time $t$ \\
\hline$\tilde{C}_{j k}$ & Spectral efficiency for specific channel \\
\hline$\tilde{C}_{M}$ & Overall spectral efficiency at the network site \\
\hline$P_{o p}$ & Operating power expenditure \\
\hline$\sigma_{e N B_{M}}$ & $\begin{array}{l}\text { Network factor that scales down from LTE macrocell to } \\
\text { the small cells }\end{array}$ \\
\hline$P_{k, e N B}$ & Power consumption due to eNB macrocell backhauling \\
\hline$P_{f c}$ & Functional power consumption \\
\hline $\mathcal{N}_{s e c}$ & Number of sectors for UEeNB \\
\hline $\mathcal{N}_{a m p}$ & Number of antenna per sector \\
\hline$\gamma_{a m p}$ & Power amplifier efficiency \\
\hline$P_{\alpha_{0}}$ & Transmit power offset \\
\hline$h_{c}$ & Cooling losses in the system \\
\hline$h_{b}$ & Battery backup losses in the system \\
\hline$P_{e N B}^{c o(k)}$ & Power consumption for the eNB macrocell \\
\hline$P_{s c}^{c o(k)}$ & Power consumption for small cells \\
\hline$\stackrel{\circ}{A}_{e N B}$ & $\begin{array}{l}\text { Coefficient account for power consumption to scale the } \\
\text { average radiated to power for the eNBs macrocell }\end{array}$ \\
\hline$\stackrel{\circ}{A s}_{s c}$ & $\begin{array}{l}\text { Coefficient account for power consumption to scale the } \\
\text { average radiated to power for small cells }\end{array}$ \\
\hline$P_{\alpha_{0}}^{e N B}$ & Transmit power offset for eNB macrocell \\
\hline$\stackrel{\alpha}{o}_{\alpha_{o}}^{s c}$ & Transmit power offset for small cell base station \\
\hline$P_{\text {site }}^{c o(M)}$ & Site power consumption \\
\hline$P_{\mathcal{A}}^{M}$ & Area power consumption \\
\hline $\mathcal{A}_{\text {site }}$ & Area site is $3 \sqrt{3} / 2\left(R_{\text {site }}\right)^{2}$ \\
\hline$R_{\text {site }}$ & Radius of the network site \\
\hline$\Pi_{k}^{\max }$ & Upper bound limit for the pilot power \\
\hline$\Pi_{k}^{k}$ min & Lower bound limit for the pilot power \\
\hline $\mathfrak{X}_{k}$ & Pilot power of base station $k$ \\
\hline$Z_{k j}$ & Base station $k$ covers aircraft $j$ \\
\hline$v_{s}^{j}$ & Mobile at position $s$ in the area of base station $k$ \\
\hline$S_{j}$ & Total number of aircraft in the area of base station $k$ \\
\hline$P_{k v_{1} j}^{p c h}$ & Base station $k$ is covering a aircraft at position $v_{1}^{j}$ \\
\hline$E_{k}$ & $\begin{array}{l}\text { Total number of vehicles in the sequence of } s \text { within } \\
\text { base station } k\end{array}$ \\
\hline$P^{*}$ & Optimum amount of pilot power \\
\hline$E_{\text {site }}$ & Site energy consumption \\
\hline $\mathcal{N}_{k}^{e N B}$ & Number of eNB macrocell base stations in the sites \\
\hline$E_{M}^{e N B}$ & Energy usage over for eNB macrocell \\
\hline$\stackrel{N}{E}$ & $\begin{array}{l}\text { Energy consumed by other aircraft network elements } \\
\text { such as the core network and radio network controllers }\end{array}$ \\
\hline
\end{tabular}




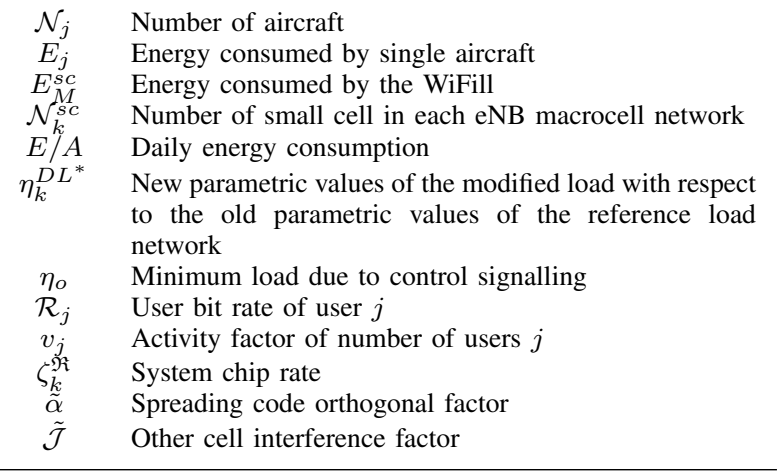

provide a flexible coverage for 5G UDNs. The configuration of radio access parameters can involve the adjustment of pilot transmission power using intelligent learning technique (e.g. Reinforcement Learning (RL)). More specifically, for example, [19] proposed an intelligent configuration of antenna power transmission in order to adjust its coverage and capacity. The authors considered distributed algorithm in macrocell network scenario and present three different learning approaches with performance evaluations. Other techniques aim to adjust the radio transmission parameters based on measurements of signal received from neighbour base stations. For example, [20] studied an energy-efficient high-capacity LTE macrocell network through utilizing small cells and energy saving strategy. However, this requires a low-latency backhaul to distribute packets between base stations and to coordinate multi-point transmission techniques [21]. It is important to understand that existing schemes are not generic and cannot be applied directly to multi-tier cells due to various differences in vendor specific physical specification, maximum transmit power for local domains, and so on. In [22], the authors investigated an energy saving scheme that exploits the amount of traffic processed by a certain cell to decide whether a cell with low load should be switched to sleep mode. Similarly, the dynamic adjustment in the number of active base stations and their coverage domains is analysed considering the traffic status throughout the network [23], [24].

In [23], the authors evaluated various cell discovery techniques tailored for energy efficient detection of small cells deployed. In ultra-dense paradigm, adjusting the power levels of the transmitted pilot signal may change the coverage area of the cells. Although the energy efficiency approach has been considered in various scenarios such as sleep mode, scheduling in LTE macrocell base station [25], and energyefficient cooperative multicast [26]. The 5G UDNs paradigm, which is considered in this study is very different, by developing an energy-efficient backhauling scheme for small cells deployment in macrocell domain, where the power transmissions are activated for lower energy consumption. This can facilitate some guidelines for the existing wireless solutions. Specifically, the adoption of pilot power approach in order to systematically address the critical issue of power saving in dense network, together with the ultra-dense capacity management solution. In summary, existing studies on the energy-efficient of 5G UDNs only consider the orthogonal channel deployment and the impact of interferences. These observations motivate us to address the power optimization problem to develop a mathematical model using linear integer programming to maximize the throughout while minimizing the overall transmitted power [27]. The airport capacity measurement is considered for Cranfield Airport environment. This power transmission optimization represent local base stations with the intention to cover large parts or the entire airport area. It is however likely that the capacity requirement in particular airport with large coverage will be too tough to be managed by one or a few base stations to cover the entire airport surface.

\section{B. Contributions}

In this paper, we provide a new formulation for the energyefficient optimization technique based on 5G UDNs, where macrocell base stations overlaid with small cells cooperatively serve multiple users using shared resources with guaranteed data rate requirements. The challenge in dense networks is to achieve high capacity with satisfaction for traffic demand. To tackle this problem, we first formulate the power optimization problem, which is critical for proposing an optimal solution that maximizes the energy efficiency while minimizing the overall transmission power. Then, we propose an intelligent pilot power allocation strategy that responds to the dynamic traffic changes based on the energy saving metric. The major contributions of this paper are outlined as follows:

1) Maximizing spectral efficiency: In any given area the achievable spectrum efficiency is impacted by the underlying user population. We provide new modelling for area spectral efficiency in dense networks loaded with different traffic profiles with the assumption that each access point transmits at maximum power to cover all users. Then, we formulate the DownLink (DL) throughput maximization problem to improve the overall system services.

2) Power optimization problem with a consideration of data traffic requirements: Most existing transmit power optimization rely on the statistics of the signal and interference power [18] and [22]. Different from this, we propose an intelligent pilot power for small cells embedded in macrocell area that addresses both power consumption reduction and cell coverage balancing. Hence, we formulate an optimization linear problem of pilot power control in order to minimizing the energy consumption by fully exploit the cell coverage and attain high throughput.

3) Simplify the power optimization problem using an enhanced integer programming method: We reduce the power consumption using energy-aware pilot power schemes, for cooperative transmission and allow a quick response to the dynamic changes in microcell traffic load. Optimizing the pilot power allows us to restructure the cell coverage area with minimal transmission power using convex theory.

4) Network capacity: We calculate the network capacity and energy efficiency for various deployment scenarios. We first develop energy performance metrics for $5 \mathrm{G}$ UDNs. In addition, we exam the potential daily energy 
savings when deploying small cells along with macrocell base stations with different values of transmitted power and different traffic load profiles.

5) Numerical analysis and simulations: We carry out extensive numerical analysis and simulations to validate the effectiveness and efficiency of our proposed power optimization approach. Theoretical analysis and simulation results show that the proposed pilot power optimization model not only has low power consumption, but also offers energy efficiency performance enhancement and increases the system resources capability.

The rest of this paper is organized as follows: the proposed system model in dense networks is presented in Section II. The analysis of site energy consumption is given in Section III. The system performance and numerical evaluation are discussed in Section IV. Conclusions are drawn in Section V. Main parameters, notations, and variables used in this paper are listed in Table I.

\section{SySTEM MODEL}

We consider $5 \mathrm{G}$ UDNs with $m=\{1, \ldots, M\}$ cells in the system. The set of base stations is denoted by $k=\{1, \ldots, K\}$. Each base station has a set of vehicles denoted by $j=$ $\{1, \ldots, J\}$. Let $P_{k}^{m x}$ be the maximum transmit power of any base station. As for a grid of base stations, $G_{k j}^{d l}$, is the power gain between a base station $k$ and the aircraft $j$. Let $P_{k}^{t o t}$ and $P_{j}^{t o t}$ denote the total power transmitted from base station $k$ and aircraft $j$, respectively. The total power in any cell $m$ in the network is given by $P_{k}^{t o t} \leq P_{k}^{m x}$ as it is completely dependent on the DL traffic. Thus, the DL load factor can be obtained as $\eta_{k}^{d l}=P_{k}^{t o t} / P_{k}^{m x}$, which is the amount of power devoted to the pilot channel for aircraft $j$ in cell $m$. We assume that all base stations operate at maximum power.

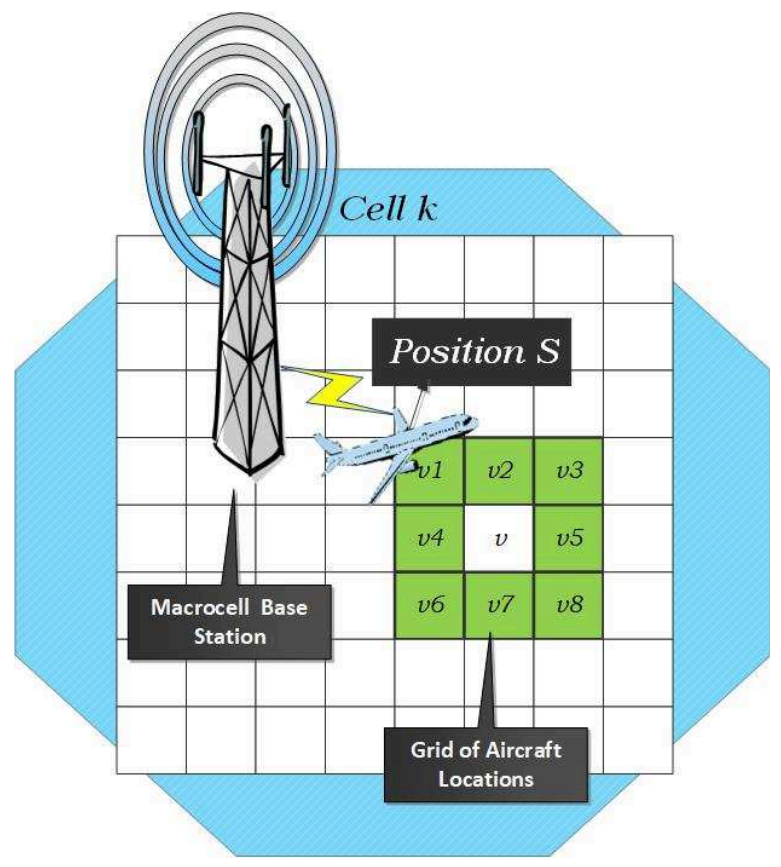

Fig. 1. Adaptive coverage and cell grid of aircraft locations.
The total interference of aircraft $j$ from its surroundings with respect to the base station $k$ is given by

$$
I_{k j}=G_{k j}^{d l} P_{k}^{t o t} \delta_{p c h}^{d l}+\sum_{j=1}^{J}\left(G_{k j}^{d l} P_{j}^{t o t} \eta_{k}^{d l}+\mathcal{N}_{j}\right),
$$

where $\mathcal{N}_{j}$ denotes the thermal noise power, and $\delta_{p c h}^{d l}$ the power interference caused by non-orthogonal signals that are transmitted by eNB.

The received signal to interference density $E_{C} / I_{O}$ ratio for aircraft $j$ from base station $k$ can be calculated as

$$
\Gamma_{k j}^{p c h}=\frac{G_{k j}^{d l} P_{k j}^{p c h}}{I_{k j}} .
$$

Thus, the updated pilot power signal can be derived as

$$
\begin{aligned}
P_{k j}^{p c h}=\frac{\Gamma_{k j}^{p c h}}{G_{k j}^{d l}}\left(G_{k j}^{d l} P_{k}^{t o t} \delta_{p c h}^{d l}+\right. & \\
& \sum_{j=1}^{J} \sum_{k=1}^{K}\left(G_{k j}^{d l} P_{j}^{t o t} \eta_{k}^{d l}+\mathcal{N}_{j}\right) .
\end{aligned}
$$

To achieve an active coverage, the pilot signal power of base station $k$, received at aircraft $j$ must fulfil the following condition

$$
G_{k j}^{d l} P_{k j}^{p c h} \geq \rho_{j}
$$

where $\rho_{j}$ is the threshold sensitivity required to detect a signal at aircraft $j$.

In the following sub-sections, we identify the metrics for area spectral efficiency and power consumption in order to solve the pilot power optimization problem.

\section{A. The Metric of Area Spectral Efficiency}

Spectral efficiency is one of the key performance parameters and major challenge in ultra-dense domain. The maximum throughput and the area spectral efficiency (ASE) of the network can be represented in $\mathrm{bps} / \mathrm{Hz} / \mathrm{km} 2$ and scale linearly according to the base stations deployments [28]. Therefore, the capacity of a link between base station $k$ and vehicle $j$ is given by

$$
C_{k j}^{d l}=\beta_{k} \log _{2}\left(1+\Gamma_{k j}^{p c h}\right)
$$

where $\beta_{k}$ is the frequency bandwidth available in the site covered by base station $k$.

As the traffic and aircraft $j$ locations are fluctuation, we use (5) to identify the interim DL capacity for the aircraft at a specific time $t$ as 


$$
C_{j}^{d l}(t)=\beta_{k} \log _{2}\left(1+\frac{P_{k j}^{p c h}(t)}{\sum_{j=1}^{J} P_{j}^{t o t}(t) \eta_{j}^{d l}(t)+\mathcal{N}_{j}}\right),
$$

where $P_{j}^{t o t}(t)$ is the power received by aircraft at time $t$.

We propose a new approach, that can be used for dynamic traffic, called interim spectral efficiency $\tilde{C}_{j k}$ for a specific channel at time $t$, given by

$\tilde{C}_{j k}(t)=\frac{C_{j}^{d l}(t)}{\beta_{k}}=\log _{2}\left(\frac{1+P_{k j}^{p c h}(t)}{\sum_{j=1}^{J} P_{j}^{t o t}(t) \cdot \eta_{j}^{d l}(t)+\mathcal{N}_{j}}\right)$.

Note that the area spectral efficiency is expressed in bits $/ \mathrm{sec} / \mathrm{Hz} / \mathrm{km}^{2}$. The overall spectral efficiency obtained at the 5G UDNs $\tilde{C}_{M}$ can be calculated as

$$
\tilde{C}_{M}=\sum_{k=1}^{K} \sum_{j=1}^{J} \tilde{C}_{j k}(t) .
$$

This spectral efficiency reflects the 5G UDN performance for a certain traffic profile.

\section{B. Throughput Maximization Problem}

The coverage of ultra-dense is defined as the probability that the signal-to-interference-noise ratio (SINR) of aircraft is above a SINR threshold [28]. Hence, We formulate the DL throughput maximization problem built up on equation (5) as

$$
\begin{gathered}
\operatorname{Max} \sum_{k=1}^{K} \sum_{j=1}^{J} \beta_{k j} \log _{2}\left(1+\Gamma_{k j}^{p c h}\right), \\
\text { s.t. } \sum_{j=1}^{J} \beta_{k j} \leq \beta_{k}, \forall_{j}, \\
\sum_{k=1}^{K} P_{k}^{t o t} \leq P_{k}^{m x}, \forall_{k}, \\
P_{k}^{t o t} \leq P^{*},
\end{gathered}
$$

where $\Gamma_{k j}^{p c h} \geq 0$ is the received signal to interference density (Ec=Io) ratio for aircraft $\mathrm{j}$ from base stations $\mathrm{k}$. The $P_{k}^{t o t} \geq 0$ is the transmission power from base station $k$ to aircraft $j$. $\beta_{k j}$ is the total bandwidth of the base station $k$ at aircraft $j$, $P^{*}$ is the optimum transmission power of base station $k$ to aircraft $j$.

\section{Power Consumption Model}

We develop a power consumption model to evaluate the consumed power for macrocell overlaid with small cells in 5G UDNs. In order to analyse the overall power savings that can be obtained in ultra-dense environment, we start by identifying the operating power expenditure (OPEX) [29] of the network, $P_{O P}$ given as

$$
P_{o p}=\sum_{k=1}^{K}\left(\eta_{k}^{d l} P_{k}^{m x} \sigma_{e N B_{M}}+P_{k, e N B}\right),
$$

where $\eta_{k}^{d l}$ denotes the cell load that may vary between 0.1 and 0.9 depending on the users capacity and radio interface configuration, $P_{k}^{m x}$ denotes the power that is required to create the mximum transmission power in the antenna output, $\sigma_{e N B_{M}}$ denotes the network factor that scales down from macrocell to the small cells, and $P_{k, e N B}$ is the power consumption due to eNB backhauling.

The macrocell base station also incorporates functional power expenditure due to hardware components cooling resulted from thermal radiation regardless of the transmit power. This becomes significant as the spectral efficiency of the DL increases, causing additional backhauling and signal processing. This functional power consumption $P_{f c}$ can be calculated in Joules/sec as

$$
\begin{aligned}
P_{f c}=\mathcal{N}_{s e c} \mathcal{N}_{a m p} & \left(\gamma_{a m p} P_{k}^{m x}+P_{\alpha_{o}}\right) \\
& +\left(1+h_{c}\right)\left(1+h_{b}\right) P_{k, e N B},
\end{aligned}
$$

where $\mathcal{N}_{\text {sec }}$ is the number of sectors for eNB, $\mathcal{N}_{a m p}$ is the number of antenna per sector, $P_{k}^{m x}$ is the transmit power of the base station, $\gamma_{a m p}$ is the power amplifier efficiency, $P_{\alpha_{O}}$ is the transmit power offset which depends on the power spent for signal processing. $h_{c}$ and $h_{b}$ respectively denote the cooling and battery backup losses in the system.

The relation between the average radiated power obtained using (10) and (11) with respect to the site's power consumption is linearly modelled for both macrocell and small cell base station sites [30], can be calculated as

$$
\begin{gathered}
P_{e N B}^{c o(k)}=\mathcal{N}_{s e c} \mathcal{N}_{a m p}\left(\AA_{e N B} P_{k}^{m x}+P_{\alpha_{O}}^{e N B}\right) \\
P_{s c}^{c o(k)}=\AA_{s c} P_{k}^{m x}+P_{\alpha_{O}}^{s c},
\end{gathered}
$$

where $\AA_{e N B}$ and $\AA_{s c}$ are the coefficients to scale the average radiated power for the macrocell and small cells respectively. $P_{\alpha_{O}}^{e N B}$ and $P_{\alpha_{O}}^{S c}$ are the transmit power offset for macrocell and small cell, respectively.

The overall site power consumption can be calculated as

$$
P_{\text {site }}^{c o(M)}=\sum_{m=1}^{M}\left(P_{e N B}^{c o(k)}+P_{s c}^{c o(k)}\right) .
$$


The area power consumption is defined as the average power consumption per cell divided by the cell area measured in Watt $/ \mathrm{Km}^{2}$. Finally, the overall area power consumption can be expressed as

$$
P_{\mathcal{A}}^{M}=\frac{P_{\text {site }}^{c o(M)}}{\mathcal{A}_{\text {site }}}
$$

where $\mathcal{A}_{\text {site }}$ is the area site is equal to $3 \sqrt{3} / 2\left(R_{\text {site }}\right)^{2}$ in $\mathrm{km}^{2}$ and $R_{\text {site }}$ is the radius of the network site.

\section{Formulation of the Pilot Power Problem}

We aim to minimize the pilot power value according to the data rate requirement of each user, where the macrocell and a set of small cell base stations jointly serve all aircraft users located in the dense networks. Therefore, we introduce a pilot power optimization problem with optimum coverage requirement, as follows

$$
\begin{gathered}
\min \sum_{j=1}^{J} P_{k j}^{p c h}, \\
\text { s.t. } G_{j k}^{d l} P_{k j}^{p c h} \geq P_{j}, \\
\sqcap_{k}^{\max } \leq P_{k j}^{p c h}, \\
0 \leq \sqcap_{k}^{\min } \leq P_{k}^{\max } .
\end{gathered}
$$

In constraint (16b) at least one base station is required to serve a set of aircraft users. Constraints (16c) and (16d) sets the upper and lower bound limit, respectively, for the pilot power in base station $k$. We use pilot power minimization as specified in [31] for constrained multi-objective optimization to formulate the cell-aircraft coverage problem. For simplicity, we do not include the traffic and service area in this model by letting them to be equal to one. The notations are used in the following formulations, $\mathfrak{X}_{k}$ is the pilot power of base station $k$, and $Z_{k j}$ is a binary variable that equals 1 if base station $k$ covers aircraft $j$ and 0 otherwise. The problem formulation for full coverage $(\mathrm{P} 1)$ is given as

$$
\begin{gathered}
\text { P1: } \min \sum_{k=1}^{K} \mathfrak{X}_{k}, \\
\text { s.t. } \sum_{k=1}^{M} Z_{k j} \geq 1, j=1, \ldots, J, \\
P_{k j}^{p c h} Z_{k j} \leq \mathfrak{X}_{k} k=1, \ldots, K, j=1, \ldots, J, \\
\prod_{k}^{m i n} \leq \mathfrak{X}_{k} \leq P_{k}^{\max } k=1, \ldots, K, \\
Z_{k j} \in\{0,1\}, k=1, \ldots, K, j=1, \ldots, J, \\
\mathfrak{X}_{k} \in\{0,1\}, k=1, \ldots, K, j=1, \ldots, J,
\end{gathered}
$$

Constraint (17a) guarantees full coverage. The pilot power level of base station $\mathrm{k}$ must be at least equal or greater than the maximum $P_{k j}^{p c h}$ value among its aircraft users as in constraint (17b). The constraint (17c) ensures that pilot power level in cell $k$ is within the given interval.

\section{E. Enhanced Formulation for Near-Optimum Solution}

In the ultra-dense domain we need to identify the optimal pilot power level for each cell. Therefore, we use the integer programming based incremental technique to find a nearoptimal solution that can be applicable to all cell sites. For base station $k$, we introduce a new sequence for $P_{k j}^{p c h}$ in ascending order using the set $v_{1}^{j}, v_{2}^{j}, \ldots, v_{s-1}^{j}, v_{s}^{j}$, where $v_{s}^{j}$ denotes the aircraft at positions in the area of base station $k$, as shown in Fig. 1.

We re-model the pilot power according to this new sequence of pilot power for base station $k$ in an incremental technique as ${ }_{P}^{p c h} \leq P_{k v_{1}^{j}}^{p c h} \leq \ldots \leq P_{k v_{s-1}^{j}}^{p c h} \leq P_{k v_{s}^{j}}^{p c h} \leq$. . Therefore, the value of $P^{p c h}$ is used by base station $k$ to cover a aircraft at position $v_{1}^{j}$. For any subsequent positions in the order of $s \in\left\{2, \ldots, E_{k}\right\}$ an additional power increment of $P_{k v_{s}^{j}}^{p c h^{J}}$ is added to the initial values in order to cover the additional aircraft distances, where $E_{k}$ is the total number of vehicles in the sequence of $s$ within base station $k$.

Let $\partial \in\{0,1\}$ denote the required level of traffic coverage $(\partial=1.0$ means full coverage $) . y_{s}$ is a binary variable that equals 1 if base station $k$ covers aircraft $j$ and 0 otherwise. Thus, we reformulate the power minimization as

$$
\begin{gathered}
\text { P2: } \min \sum_{k=1}^{K}\left[\eta_{k}^{m i n}+\sum_{s=1}^{E_{k}} P_{k v_{s}^{j}}^{p c h^{J}}{ }_{k v_{s}^{j}}\right], \\
\text { s.t. } \sum_{k=1}^{K} f_{s} y_{s} \geq \partial F, \\
\sum_{k=1}^{K} Z_{k j} \geq y_{s}, k=1, \ldots, K, \\
\leq \mathfrak{X}_{k v_{s}^{j}}^{j}, k=1, \ldots, K, j=1, \ldots, E_{k}, \\
Z_{k j} \in\{0,1\}, k=1, \ldots, K, j=1, \ldots, J, \\
y_{s} \in\{0,1\}, j=1, \ldots, J,
\end{gathered}
$$

In $\mathrm{P} 2$, constraints $(18 a)$ referred to the base station pilot constraint, states that if the base station $k$ covers any aircraft 
$j$, then the pilot power equals $P_{k v_{s}^{j}}^{p c h}$. Constraints (18b) define the minimum uniform pilot power that satisfies the coverage requirement. The new sequence of pilot power for base station $k$ in an incremental technique is stated in (18c). It is important to note that the base station with pilot power level equal or close to $\Pi_{k}^{\min }$ may consider to switch off [32]. Therefore, the pilot power of the base station is the required minimum $\sqcap_{k}^{\min }$ if it covers at least one aircraft, otherwise it is zero.

\section{Site ENERgy CONSUMPtion}

In this section, we adopted an energy consumptions model as specified in [33] to calculate the energy saving obtained from various traffic profiles with the area power efficiency. The energy consumption for a network site using real time application can be estimated at a certain time interval $t$ as

$$
E_{\text {site }}=\sum_{m=1}^{M} \sum_{k=1}^{K}\left[\mathcal{N}_{k}^{e N B}\left(P_{o p}+\left(\eta_{k}^{d l} P_{k}^{m x}\right)\right) t\right]
$$

where $\mathcal{N}_{k}^{e N B}$ refers to the number of macrocell base station in the site. The energy usage over time $t$ (seconds) for an 5G UDN can be calculated as

$$
E_{M}^{e N B}=\sum_{k=1}^{M} \mathcal{N}_{k}^{e N B} E_{s} i t e+\sum_{j=1}^{J} \mathcal{N}_{j} E_{j}+E_{O} .
$$

This is a standard definition for the energy consumed in all base stations in the network. Here $E_{\text {site }}$ is the energy consumed by a single base station site, $\mathcal{N}_{j}$ refers to the number of aircraft, $E_{j}$ refers to the energy consumed by a single aircraft, and $E_{O}$ is the energy consumed by other aircraft network elements, such as the controllers, core, and radio access network.

When small cell systems are deployed in 5G UDN, the energy consumed by the small cell network can be expressed as

$$
E_{M}^{s c}=\sum_{k=1}^{K} \mathcal{N}_{s} \text { ite } \mathcal{N}_{k}^{e} N B+\left(\mathcal{N}_{k}^{s c} \cdot P_{s c}^{c o(k)}\right) t,
$$

where $\mathcal{N}_{k}^{s c}$ is the number of small cells in each macrocell domain and $P_{S c}^{c o(k)}$ is the power usage of the small cell base station over time $t$.

We develop an analytical model with intelligent coverage area and dynamic traffic to measure the network performance, expressed in terms of the daily energy consumption per square kilometer $\left(\mathrm{kWh} / \mathrm{km}^{2}\right)$ as follows

$$
\begin{aligned}
&\left(E_{M}^{e N B}, E_{M}^{s c}\right)_{\text {Area }} \\
&=\sum_{k=1}^{K}\left[\left(\mathcal{N}_{\text {site }}^{n} \mathcal{N}_{k}^{e N B} \frac{P_{o p}+\left(\eta_{k}^{d l^{*}} P_{k}^{m x}\right)}{\mathcal{N}_{\text {site }} \mathcal{A}_{\text {site }}}\right)\right. \\
&\left.\quad+\left(\frac{\mathcal{N}_{k}^{s c} P_{s c}^{c o}(k)}{\mathcal{A}_{\text {site }}}\right)\right] 24 .
\end{aligned}
$$

The number of sites in the new deployment is represented by $N_{\text {site }}^{n}$ and the corresponding load $\eta_{k}^{d l^{*}}$ refers to the new parametric values of the modified load network with respect to the old parametric values of the reference load network.

To simplify calculations, we assume a fixed site as stated in [34] with a dynamic traffic in order to measure the impact of traffic, and daily energy consumption changes is given by

$$
\begin{aligned}
\left(E_{M}^{e N B}, E_{M}^{s c}\right)_{\text {Area }} & \\
& =\sum_{k=1}^{K}\left[\mathcal{N}_{k}^{e N B} \frac{P_{o p}+\left(\eta_{k}^{d l^{*}} P_{k}^{m x}\right)}{\mathcal{N}_{\text {site }} \mathcal{A}_{\text {site }}}\right] 24 .
\end{aligned}
$$

Finally, we include the impacts of incorporating the small cells in the macrocell domain for a dynamic traffic profile as follows

$$
\begin{aligned}
\left(E_{M}^{e N B}, E_{M}^{s c}\right)_{\text {Area }} & \\
& =\sum_{k=1}^{K}\left[\left(\mathcal{N}_{k}^{e N B} \frac{P_{o p}+\left(\eta_{k}^{d l} P_{k}^{m x}\right)}{\mathcal{A}_{\text {site }}}\right)+\right. \\
& \left.\left(\frac{\mathcal{N}_{k}^{s c} P_{s c}^{c o}(k)}{\mathcal{A}_{\text {site }}}\right)\right] 24 .
\end{aligned}
$$

The focus is on the downlink of macrocell base station where the same resources are allocated. The load factor can be approximated by its average value across the cell being formulated as in [35] as

$$
\eta_{k}^{d l^{*}}=\eta_{o}+\mathcal{N}_{j} \frac{\Gamma_{k j}^{p c h} \mathcal{R}_{j} v_{j}}{\zeta_{k}^{R}}(1-\tilde{\alpha}+\tilde{\mathcal{J}}),
$$

where, $\eta_{O}$ denotes to the minimum load due to control signaling, $v_{j}$ denotes the activity factor of number of users $j, \mathcal{R}_{j}$ denotes the user bit rate, $\zeta_{k}^{R}$ denotes the system chip rate, $\tilde{\alpha}$ denotes the spreading code orthogonally factor and $\tilde{\mathcal{J}}$ is represent other to own cell interference factor. The load is considered to be dependent on the expected $\tilde{\alpha}$ and $\tilde{\mathcal{J}}$ over the entire cell area, where the system characteristics are listed in Table II.

The relation between energy consumption in $\mathrm{kWh}$ per $\mathrm{km}^{2}$ and the load from (0.1 to 1$)$ is displayed in Fig. 2 . It is observed that when the small cells are deployed with 
TABLE II

SYSTEM CHARACTERISTICS.

\begin{tabular}{ll}
\hline \hline Parameter & Value \\
\hline System chip rate & $3.81 \mathrm{Mcps}$ \\
Shadow fading margin & $6 \mathrm{~dB}$ \\
Mean $\tilde{\alpha}$ (Average orthogonality factor in the cell) & 0.45 \\
Mean $\tilde{\mathcal{J}}$ interference ratio & 0.60 \\
Activity factor $a_{j}$ & 1.00 \\
Minimum load & 0.075 \\
System load & 1 to $100 \%$
\end{tabular}

total power $(6 \mathrm{~W}, 3 \mathrm{~W}$, and $2 \mathrm{~W})$ in macrocell domain, around $25 \%, 17 \%$, and $11 \%$ of energy would be saved and it keeps on increasing as the number of small cells increases. The deployment of small cells results in reducing the load and power delivered through the macrocell. Another observation is that for the low power case, the energy saving keep decreasing with increasing the traffic load in the system.

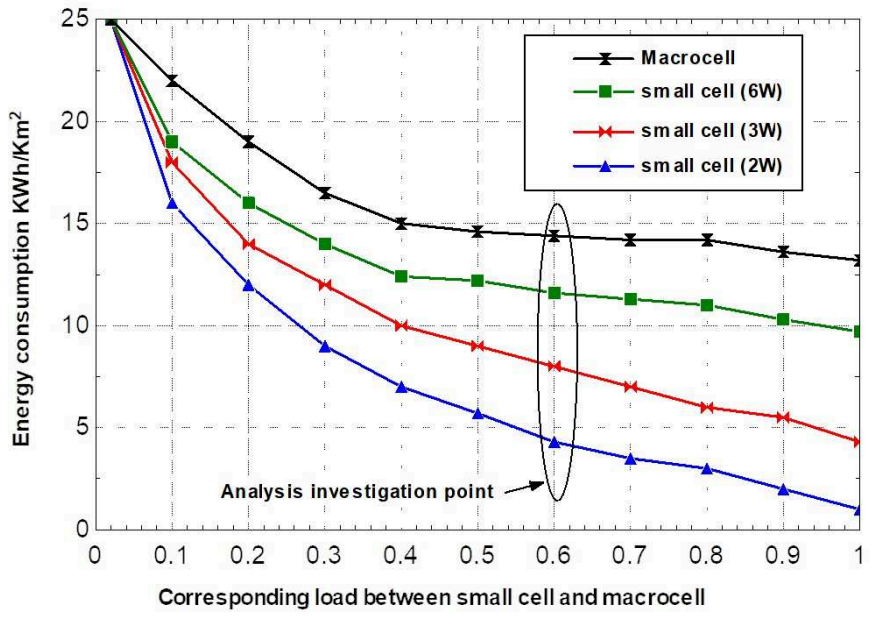

Fig. 2. Energy consumption vs. ratio connection of macrocell and small cell.

Numerical results reveal that for current network design and operation, the energy efficiency is mostly independent of the traffic load. This highlights the significant potential for energy savings by improving the energy efficiency of base stations at low load. Furthermore, the results prove that small cell link can effectively save the spectrum resources for the macrocell subscribers.

\section{A. Numerical Analysis}

In this section, we evaluate the energy saving performance and the efficiency tradeoff for the proposed power consumption model of multiple cellular communication domains. The numerical analysis of system networks is presented to obtain the optimum inter site distance (ISD) value. Our scenario has considered seven macrocell with variable grid of sites symbolized by the ISD, which ranges between 500-2000 meters. We assume that the cell area is $95 \%$ covered for macrocell and small cells domain to get better coverage performance. Mobile station terminals are placed randomly in the macrocell domain, small cell sites are assumed to support a circular area of radius $30 \mathrm{~m}$, which are positioned at the cell edges where, the signal levels of the macrocell is expected to be low. The spectral efficiency $\tilde{C}_{M}$ is assumed to be $6 \mathrm{bit} / \mathrm{s} / \mathrm{Hz}$ according to (8). The power model parameters are selected as the values resulting from (13) and (14), assuming that the coefficients of power consumption are $\AA_{e N B}=3.8, P_{\alpha_{O}}^{e N B}=68.8 \mathrm{~W}, \AA_{s c}$ $=6.3, P_{\alpha_{O}}^{S c}=0.5 \mathrm{~W}$. The power consumption is calculated according to (15). The SINR requirements according to [36] for transmission bandwidth are shown in Table III below.

TABLE III

THE REQUIREMENTS OF MACROCELL SINR

\begin{tabular}{clccc}
\hline \hline CQI & Modulation & $\begin{array}{c}\text { Code } \\
\text { Rate }\end{array}$ & $\begin{array}{c}\text { Spectral Efficiency } \\
{[\mathrm{bps} / \mathrm{Hz}]}\end{array}$ & $\begin{array}{c}\text { Receiver } \\
\text { SINR [dB] }\end{array}$ \\
\hline 0 & & & & \\
1 & QPSK & $1 / 5$ & 0.40 & -2.9 \\
2 & QPSK & $1 / 5$ & 0.40 & -2.9 \\
3 & QPSK & $1 / 4$ & 0.55 & -1.7 \\
4 & QPSK & $1 / 3$ & 0.66 & -1.00 \\
5 & QPSK & $1 / 2$ & 1.00 & 2.0 \\
6 & QPSK & $2 / 3$ & 1.33 & 4.3 \\
7 & QPSK & $3 / 4$ & 1.50 & 5.5 \\
8 & QPSK & $4 / 5$ & 1.60 & 6.2 \\
9 & 16-QAM & $1 / 2$ & 2.00 & 7.9 \\
10 & 16-QAM & $2 / 3$ & 2.66 & 11.3 \\
11 & 16-QAM & $3 / 4$ & 3.00 & 12.3 \\
12 & 16-QAM & $4 / 5$ & 3.20 & 12.8 \\
13 & 16-QAM & $2 / 3$ & 4.00 & 15.3 \\
14 & 16-QAM & $3 / 4$ & 4.50 & 17.5 \\
15 & 16-QAM & $4 / 5$ & 4.80 & 18.6 \\
\hline
\end{tabular}

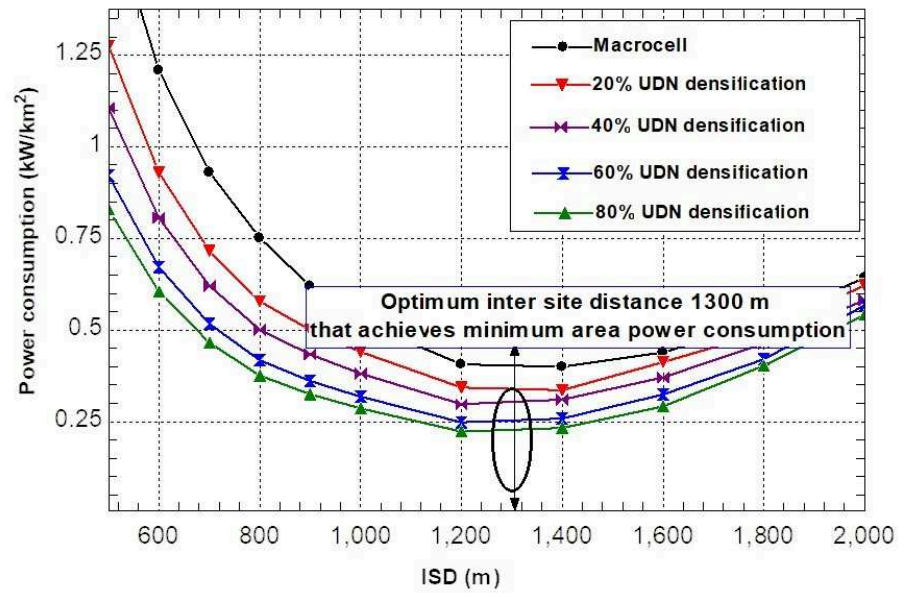

Fig. 3. Area power consumption vs. ISD $\left(\beta_{k}=2 \mathrm{GHz}, \rho_{j}=-70 \mathrm{dBm}\right)$.

The area power consumption analysis as a function of ISD for various small cell deployment densities is depicted in Fig. 3. As expected, results show that the area power consumption decreases as the small cell density increases compared to the case when only macrocell unit is in place. Also, all power consumptions decrease with the increase of ISD. This confirms the fact that the small cells deployment decreases the energy consumed in the system by utilizing the smaller transmission domains and shorter formulated links. By ignoring the impact of shadowing, it is easier to identify the optimum cell radius as well as the optimum ISD that achieve minimum area power consumptions for each analyzed scenario. Fig. 3. shows that the optimum corresponding ISD is $1300 \mathrm{~m}$ achieving the minimum area power consumption of $400 \mathrm{~W} / \mathrm{km}^{2}$. When small cell nodes are deployed, they 
offload the premium users from the macro base station and free macro resources to serve basic users. It can be observed, that all deployment strategies have the same optimal ISD but with different values of minimum power consumptions. For ultra-dense deployment scenarios as percentage of $20 \%$, $40 \%, 60 \%$, and $80 \%$ of small cells in the macrocell domain. The power consumptions range between $800-1270 \mathrm{~W} / \mathrm{km}^{2}$, which is considerably less than the power consumed in the traditional services delivered by the macrocell base station. Thus, the small cells deployment reduces the power consumed in the system. However, small cell base stations are not able to increase the optimum inter site distance for the site under coverage. It is clear that the new model improves the area power consumption with a logical and applicable approach by using optimization power model. The power consumption for higher densities of deployed small cell is decreasing for the same ISD value. The lowest power for ISD occurs at $1300 \mathrm{~m}$ for the macrocell domain.

\section{SYSTEM PERFORMANCE}

We carry out a system simulation level to evaluate the performance of 5G UDNs overlaid small cells. The simulated network incorporates seven macrocells, where the base station is placed at cell centre of hexagonal layout with a fixed ISD and number of small cells are distributed randomly along the edge of the cells. Each macrocell includes three sectors with antenna pointing in the three horizontal directions separated by 120 degrees. In this radio system, the estimation of load demand fluctuation and configuration is considered to show how it affects the energy consumption level. The macrocell has an optimum value of ISD $=1.3 \mathrm{~km}$ obtained from our numerical calculations. The small cell radius is assumed to be $30 \mathrm{~m}$, for a cell range of $R_{k}$, the cell area can be calculated as $3 \sqrt{3} / 2\left(R_{k}\right)^{2}$. The small radio systems are deployed to compare four different network scenarios and assess how the change in the 5G UDN configuration affect the energy consumption in the wireless system. The simulation parameter values are given in Table IV.

In the simulation model, the compute the expected execution time will be each of the five min programs. The task-level performance information is returned by the execution time block, in our case is five mints. The first output port shows the total time of execution of each base rate step of the entire model.

\section{2) Impact of Traffic Load}

Fig. 4 shows the power consumption for aggregate the data traffic loads of high and low profiles. The incorporation of the new proposed scheme shows a major improvement in the system performance by reducing the values of power consumption. This is due to the fact that the optimization of pilot power creates effective coverage areas that identify the aircraft location, which means there is no requirement to transmit power beyond the actual user location. The figure shows that high traffic profile consume more power than the low traffic. This is due to the fact that more packets need high power during transmission operation compared with low traffic profiles. Results also indicate that the load performance is effected by the number of deployed small cells as this will change the traffic volume transfers a cross the networks. Therefore, more network stability and throughput advantages can be achieved with the small cell deployment. As the structure of the network changes with more deployed small cells, the overall consumed power increases gradually due to the direct impact from the increased traffic.

TABLE IV

Simulation PARAMETERS.

\begin{tabular}{ll}
\hline \hline Parameters & Value \\
\hline Simulation time, $t$ & $5 \mathrm{~min}$ \\
Transmit power of macrocell & $40 \mathrm{dBm}$ \\
Transmit power of small cell base station & $20 \mathrm{dBm}$ \\
Bandwidth & $5 \mathrm{MHz}$ \\
Antenna gain of macro/ small cell /aircraft & $(14 / 5 / 0) \mathrm{dB}$ \\
Thermal noise density & $-174 \mathrm{dBm} / \mathrm{Hz}$ \\
Noise figure in aircraft terminal & $9 \mathrm{~dB}$ \\
Antenna pattern & $\mathrm{Omni}$ \\
Propagation model of macro and small cell & $4 \mathrm{~dB}, 8 \mathrm{~dB}$ \\
Noise density & $-80 \mathrm{dBm} / \mathrm{Hz}$ \\
Path loss outdoor & $70 \mathrm{~dB}$ \\
\hline
\end{tabular}

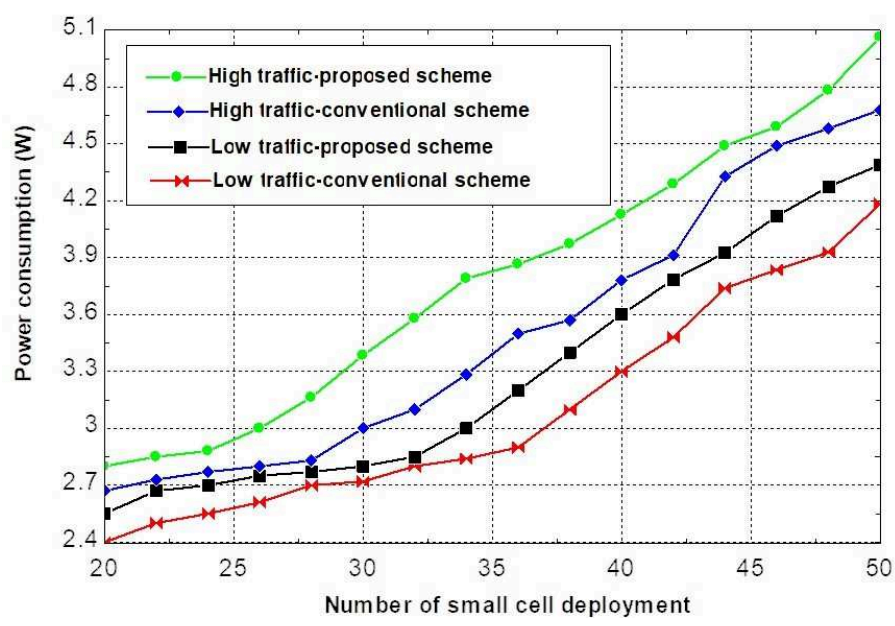

Fig. 4. Power consumption as a function of traffic.

\section{4) SINR of DL Channel}

For channel capacity, the simulation environment is introduced for modeling radio channels with line-of-sight (LoS) and non-line-of-sight (NLOS) connection between aircraft and marcocell bastion in an operating airport telecommunications system. In general, wireless communications at large airports will experience the most areas of multipath fading and (NLOS). In the simulation model, the compute the expected execution time will be each of the five min programs. The tasklevel performance information is returned by the execution time block, in our case is five mints. The first output port shows the total time of execution of each base rate step of the entire model. Fig. 5 shows the DL channel SINR of 5G UDNs for different system connection times. The graph shows the improved performance of using intelligent pilot power scheme compared to the conventional pilot power [18]. In fact, the proposed intelligent power scheme needs some time to adjust the power of transmission while identifying the user locations. 
This means that the same process of finding the user positions and adjusting power at small cells is needed again before the performance stabilizes, as shown for the interval from 70 to 240 seconds.

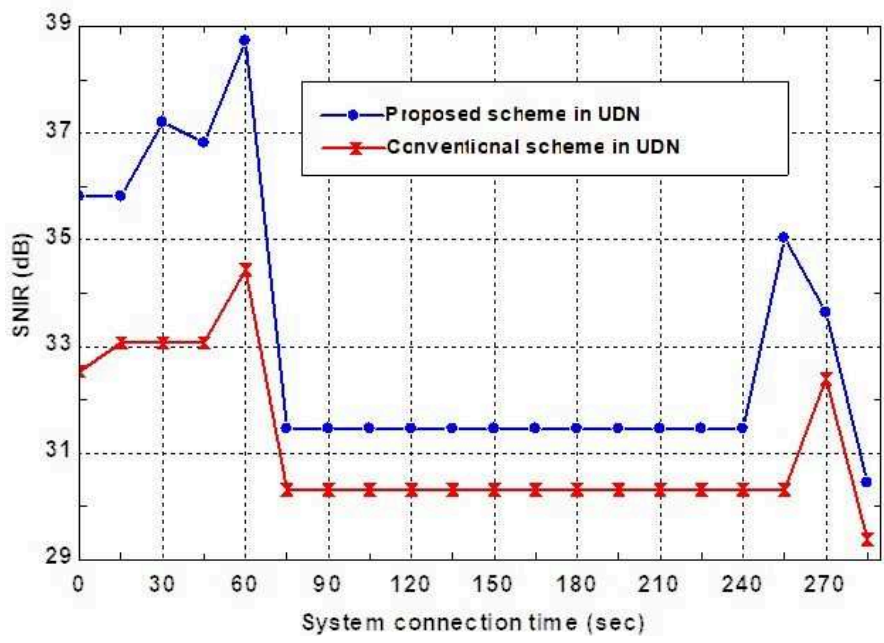

Fig. 5. SINR improvements in 5G UDN.

\section{CONCLUSIONS}

In this paper, the utilization of airport traffic load capacity problem and energy-efficiency were investigated for 5G UDNs environments employing licenced and unlicensed bands. First, we defined new optimization metrics to improve the network coverage control by leveraging an intelligent pilot power technique that allows scaling the system capacity based on fluctuations in user demands. Then, we formulated the optimization problem using linear programming method to minimize the power consumption in the ultra-dense paradigm. The performance metrics for capacity were characterized using different load profiles for the simulated dense network scenarios to validate the proposed solutions. In addition, we evaluated the optimization solution to show the potential energy savings with daily variances of both transmitted power and load profiles. Our numerical analysis demonstrated a significant savings of approximately $17 \%$ in the overall energy consumption of dense networks. Also, the system-level analysis validated the performance of intelligent power transmissions in improving the air interface with aircrafts at very low latencies. Finally, the proposed scheme of linear optimization resulted in minimized pilot power while maximized the overall system capacity for a fully autonomous and intelligent connectivity.

\section{REFERENCES}

[1] H. Chien, Y. Lin, C. Lai, and C. Wang, "End-to-End Slicing with Optimized Communication and Computing Resource Allocation in Multi-Tenant 5G Systems," IEEE Transactions on Vehicular Technology, vol. 69, no. 2, pp. 2079-2091, Feb 2020.

[2] J. Huang, Q. Xu, J. Tian, and Y. Yan, "A Dynamical Programmingbased Method to Generate Control Strategy for Air Traffic Flow," pp. 5885-5890, June 2019.

[3] G. I. Tsiropoulos, A. Yadav, M. Zeng, and O. A. Dobre, "Cooperation in 5G HetNets: Advanced Spectrum Access and D2D Assisted Communications," IEEE Wireless Communications, vol. 24, no. 5, pp. 110-117, October 2017.
[4] G. Fodor, H. Do, S. A. Ashraf, R. Blasco, W. Sun, M. Belleschi, and L. Hu, "Supporting Enhanced Vehicle-to-Everything Services by LTE Release 15 Systems," IEEE Communications Standards Magazine, vol. 3, no. 1, pp. 26-33, March 2019.

[5] A. Al-Dulaimi, S. Al-Rubaye, Q. Ni, and E. Sousa, "5G Communications Race: Pursuit of More Capacity Triggers LTE in Unlicensed Band," IEEE Vehicular Technology Magazine, vol. 10, no. 1, pp. 43-51, March 2015.

[6] H. Zhang, Y. Dong, J. Cheng, M. J. Hossain, and V. C. M. Leung, "Fronthauling for 5G LTE-U Ultra Dense Cloud Small Cell Networks," IEEE Wireless Communications, vol. 23, no. 6, pp. 48-53, December 2016.

[7] A. Al-Dulaimi, S. Al-Rubaye, J. Cosmas, and A. Anpalagan, "Planning of Ultra-Dense Wireless Networks," IEEE Network, vol. 31, no. 2, pp 90-96, March 2017.

[8] Y. S. Soh, T. Quek, M. Kountouris, and H. Shin, "Energy Efficient Heterogeneous Cellular Networks," Selected Areas in Communications, IEEE Journal on, vol. 31, no. 5, pp. 840-850, May 2013.

[9] D. Calabuig, S. Barmpounakis, S. Gimenez, A. Kousaridas, T. R Lakshmana, J. Lorca, P. Lunden, Z. Ren, P. Sroka, E. Ternon, V. Venkatasubramanian, and M. Maternia, "Resource and Mobility Management in the Network Layer of 5G Cellular Ultra-Dense Networks," IEEE Communications Magazine, vol. 55, no. 6, pp. 162-169, 2017.

[10] L. Pei, J. Huilin, P. Zhiwen, and Y. Xiaohu, "Energy-Delay Tradeoff in Ultra-Dense Networks Considering BS Sleeping and Cell Association," IEEE Transactions on Vehicular Technology, vol. 67, no. 1, pp. 734-751, Jan 2018.

[11] P. V. Klaine, M. A. Imran, O. Onireti, and R. D. Souza, "A Survey of Machine Learning Techniques Applied to Self-organizing Cellular Networks," IEEE Communications Surveys Tutorials, vol. 19, no. 4, pp. 2392-2431, July 2017.

[12] O. Onireti, A. Zoha, J. Moysen, A. Imran, L. Giupponi, M. A. Imran, and A. Abu-Dayya, "A Cell Outage Management Framework for Dense Heterogeneous Networks," IEEE Transactions on Vehicular Technology, vol. 65, no. 4, pp. 2097-2113, April 2016.

[13] K. M. S. Huq, S. Mumtaz, J. Bachmatiuk, J. Rodriguez, X. Wang, and R. L. Aguiar, "Green HetNet CoMP: Energy Efficiency Analysis and Optimization," IEEE Transactions on Vehicular Technology, vol. 64, no. 10, pp. 4670-4683, Oct 2015.

[14] S. Al-Rubaye, A. Al-Dulaimi, and J. Cosmas, "Pilot Power Optimization for Autonomous Femtocell Networks," in Wireless Advanced (WiAd), 2011, June 2011.

[15] J. Huang and V. Krishnamurthy, "Transmission Control in Cognitive Radio as a Markovian Dynamic Game: Structural Result on Randomized Threshold Policies," Communications, IEEE Transactions on, vol. 58, no. 1, pp. 301-310, January 2010.

[16] H. Zhu, T. Buot, R. Nagaike, and S. Harmen, "Load Balancing in WCDMA Systems by Adjusting Pilot Power," in Wireless Personal Multimedia Communications, 2002. The 5th International Symposium on, vol. 3, October 2002, pp. 936-940 vol.3.

[17] S. Samarakoon, M. Bennis, W. Saad, M. Debbah, and M. Latvaaho, "Ultra Dense Small Cell Networks: Turning Density into Energy Efficiency," IEEE Journal on Selected Areas in Communications, May 2016.

[18] H. Zhang, Y. Chen, Z. Yang, and X. Zhang, "Flexible Coverage for Backhaul-Limited Ultra-Dense Heterogeneous Networks: Throughput Analysis and eta-Optimal Biasing," IEEE Transactions on Vehicular Technology, vol. PP, no. 99, pp. 1-1, 2018.

[19] R. Razavi, S. Klein, and H. Claussen, "Self-Optimization of Capacity and Coverage in LTE Networks using a Fuzzy Reinforcement Learning Approach," in 21st Annual IEEE International Symposium on Personal, Indoor and Mobile Radio Communications, Sep. 2010, pp. 1865-1870.

[20] F. Richter, A. Fehske, and G. Fettweis, "Energy Efficiency Aspects of Base Station Deployment Strategies for Cellular Networks," in Vehicular Technology Conference Fall (VTC 2009-Fall), 2009 IEEE 70th, Sep. 2009.

[21] M. Sawahashi, Y. Kishiyama, A. Morimoto, D. Nishikawa, and M. Tanno, "Coordinated Multipoint Transmission/Reception Techniques for LTE-Advanced Coordinated and Distributed MIMO," Wireless Communications, IEEE, vol. 17, no. 3, pp. 26-34, June 2010.

[22] H.-S. Jo, C. Mun, J. Moon, and J.-G. Yook, "Self-Optimized Coverage Coordination in Femtocell Networks," Wireless Communications, IEEE Transactions on, vol. 9, no. 10, pp. 2977-2982, October 2010.

[23] J. Liu, Y. Kawamoto, H. Nishiyama, N. Kato, and N. Kadowaki, "Device-to-Device Clommunications Achieve Efficient Load Balancing in LTE-Advanced Networks, year $=2014$, month $=$ April, volume $=21$, number $=2$, pages $=57-65$, , Wireless Communications, IEEE. 
[24] J. Kwak, K. Son, Y. Yi, and S. Chong, "Greening Effect of SpatioTemporal power Sharing Policies in Cellular Networks with Energy Constraints," Wireless Communications, IEEE Transactions on, vol. 11, no. 12 , pp. 4405-4415, December 2012.

[25] J. Lee, N. Dinh, G. Hwang, J. K. Choi, and C. Han, "Power-Efficient Load Distribution for Multihomed Services with Sleep Mode Over Heterogeneous Wireless Access Networks," Vehicular Technology, IEEE Transactions on, vol. 63, no. 4, pp. 1843-1854, May 2014.

[26] Y. Zhou, H. Liu, Z. Pan, L. Tian, and J. Shi, "Spectral and Energyefficient Two-stage Cooperative Multicast for LTE-Advanced and Beyond," Wireless Communications, IEEE, vol. 21, no. 2, pp. 34-41, April 2014.

[27] N. Schneckenburger, T. Jost, M. Walter, G. del Galdo, D. W. Matolak, and U. Fiebig, "Wideband AirGround Channel Model for a Regional Airport Environment," IEEE Transactions on Vehicular Technology, vol. 68, no. 7, pp. 6243-6256, July 2019.

[28] J. G. Andrews, F. Baccelli, and R. K. Ganti, "A Tractable Approach to Coverage and Rate in Cellular Networks," IEEE Transactions on Communications, vol. 59, no. 11, pp. 3122-3134, November 2011.

[29] Y. Kim, T. Kwon, and D. Hong, "Area Spectral Efficiency of Shared Spectrum Hierarchical Cell Structure Networks," Vehicular Technology, IEEE Transactions on, vol. 59, no. 8, pp. 4145-4151, October 2010.

[30] F. Richter and G. Fettweis, "Cellular Mobile Network Densification Utilizing Micro Base Stations," in Communications (ICC), 2010 IEEE International Conference on, May 2010.

[31] Y. Zhang, Y.-Y. Li, E. Sousa, and Q. Zhang, "Pilot Power Minimization in HSDPA Femtocells," in Global Telecommunications Conference (GLOBECOM 2010), 2010 IEEE, Dec 2010, pp. 1-5.

[32] S. Boyd and L. Vandenberghe, Convex Optimization. Cambridge, UK: Cambridge Univ. Press, 2004.

[33] H. Holma and A. Toskala, WCDMA for UMTS. Chichester, UK: John Wiley Ltd, 2004.

[34] D. Nguyen, L.-N. Tran, P. Pirinen, and M. Latva-aho, "Precoding for Full Duplex Multiuser MIMO Systems: Spectral and Energy Efficiency Maximization," Signal Processing, IEEE Transactions on, vol. 61, no. 16, pp. 4038-4050, Aug 2013.

[35] H. Holma and A. Toskala, LTE for UMTS: OFDMA and SC-FDMA based Radio Access. Chichester, UK: John Wiley Ltd, 2009.

[36] S. Sesia, I. Toufik, and M. Baker, The UMTS Long Term Evolution. UK: John Wiley Ltd, 2009.

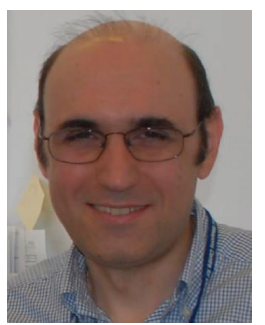

Antonios Tsourdos Antonios Tsourdos obtained an MEng on Electronic, Control and Systems Engineering from the University of Sheffield (1995), an MSc on Systems Engineering from Cardiff University (1996) and a PhD on Nonlinear Robust Flight Control Design and Analysis from Cranfield University (1999). He is a Professor of Autonomous Systems and Control with Cranfield University. He was appointed Head of the Autonomous Systems Group in 2007, Head of the Centre of Autonomous and Cyber-Physical Systems in 2012 and Director of Research - Aerospace, Transport and Manufacturing in 2015. Professor Tsourdos was member of the Team Stellar, the winning team for the UK MoD Grand Challenge (2008) and the IET Innovation Award (Category Team, 2009). Professor Tsourdos is chair of the IFAC Technical Committee on Aerospace Control, and member of the UK Autonomous Systems National Technical Committee. He is editorial board member for the IEEE Transactions on Aerospace and Electronic Systems, the Proceedings of the Institution of Mechanical Engineers, Part G: Journal of Aerospace Engineering, the Aerospace Science and Technology, the International Journal of Systems Science and the Journal of Intelligent and Robotic Systems.

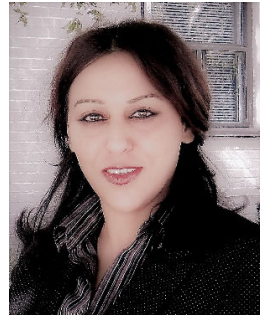

Saba Al-Rubaye Saba Al-Rubaye received her $\mathrm{Ph} . \mathrm{D}$. degree in Electrical and Electronic Engineering from Brunel University London. Currently, she is working as a Senior Lecturer and leading connected system research group in the School of Aerospace, Transport and Manufacturing at Cranfield University. She has more than 17 years of professional experience in the industry and academia with demonstrated track record of launching innovative solutions in design, testing, consultation, leadership, and program development. Dr. Saba Al-Rubaye has led and managed several research and industrial projects from conception to completion sponsored by Natural Sciences and Engineering Research Council of Canada (NSERC), Ryerson University, and industrial grants in USA. Dr. Saba Al-Rubaye is participating in developing industry standards by being an active group member of IEEE P1920.2, Standard for Vehicle to Vehicle Communications for Unmanned Aircraft Systems and of IEEE P1932.1 standard of License/unlicensed Interoperability. Dr. Al-Rubaye has published many papers in IEEE journals and conferences and a recipient of the best technical paper award twice published in IEEE Vehicular Technology in 2011 and 2015, respectively. She has been a general co-chair, TPC co-chair and has held other leading roles for many international conferences. Her main research interests focusing on airport connectivity, UAV, 5G technology, communications networks and autonomous system. Dr. Saba Al-Rubaye is registered as a Chartered Engineer (CEng) by British Engineering Council and member of the Institute of Engineering and Technology (IET), and Senior Member of Institute of Electrical and Electronics Engineers (IEEE). 
2020-06-08

\title{
Airport connectivity optimization for $5 \mathrm{G}$ ultra-dense networks
}

\author{
Al-Rubaye, Saba
}

IEEE

Al-Rubaye S, Tsourdos A. (2020) Airport connectivity optimization for 5G ultra-dense networks.

IEEE Transactions on Cognitive Communications and Networking, Volume 6, Issue 3,

September 2020, pp. 980-989

https://doi.org/10.1109/TCCN.2020.3000509

Downloaded from Cranfield Library Services E-Repository 